\title{
LOCAL COHOMOLOGY OF SEGRE PRODUCT TYPE RINGS
}

\author{
PAUL C. ROBERTS \\ Dedicated to Hans-Bjørn Foxby on the occasion of his 65th Birthday
}

\section{INTRODUCTION}

The aim of this paper is to investigate properties of the local cohomology of rings of mixed characteristic that are analogous to Segre products of rings defined over a field. The main question is whether the local cohomology can be almost killed in a finite extension (we define what this means below). There are two reasons for considering this type of ring. First, there are special properties of these rings that make it possible to answer this question. Second, and perhaps more important, Segre products are a large source of normal non-Cohen-Macaulay domains; in fact, many examples of such rings that are defined by other means turn out to be Segre products. A theorem of Goto and Watanabe [1] gives a formula for the local cohomology of the Segre product in terms of the local cohomology of the factors; this both gives a method for constructing examples of normal rings with local cohomology in given degrees and provides a method for analyzing the questions we are considering.

The general question behind this research is whether the absolute integral closure $R^{+}$of a normal domain $R$ that is either complete local or of finite type over a field is almost Cohen-Macaulay. The ring $R^{+}$is known to be Cohen-Macaulay in positive characteristic by results of Hochster and Huneke [4] and Huneke and Lyubeznik [5]. For rings of mixed characteristic, which is the most interesting case since many of the homological conjectures are open in that case (see for example Hochster [3]), R. Heitmann [2] showed that $R^{+}$is almost Cohen-Macaulay in dimension three. The higher dimensional case, as well as the question of whether $R^{+}$is Cohen-Macaulay in dimension 3, are still open. In the equicharacteristic case, there are some examples where elements of local cohomology can be almost killed in Roberts, Singh and Srinivas [10], but the general situation is not known.

In this paper we describe some examples of non-Cohen-Macaulay rings that arise from Segre products and show that the elements of local cohomology that make the ring non-Cohen-Macaulay can be almost killed in $R^{+}$.

\section{Rings of SEgRe product type}

We first recall some basic facts about ordinary Segre products. Let $k$ be a field, and let $R$ and $S$ be finitely generated graded rings over $k$. We will assume that graded rings are graded over the nonnegative integers, and they may or may not be generated over $k$ by finitely many elements of degree one. The Segre product of $R$ and $S$, denoted $R \# S$, is defined to be the subring of the tensor product $R \otimes S$ generated by elements $r \otimes s$, where $r$ and $s$ are homogeneous of the same degree. We denote an element $r \otimes s$ of this type by $r \# s$. The terminology, of course, comes from the fact that this ring arises in Segre's construction of the product of projective varieties. We can write $R \# S=\oplus_{i \geq 0} R_{i} \otimes S_{i}$.

If $M$ and $N$ are a graded $R$-module and a graded $S$-module respectively (not necessarily finitely generated), we define $M \# N$ similarly to be the sub- $R \# S$ module of $M \otimes_{k} N$ generated by the elements $m \otimes n$ where $m$ and $n$ are homogeneous of the same degree.

We list some basic properties of Segre products; these can be found in Goto and Watanabe [1]. $R \# S$ is itself a graded ring, where the degree of $r \# s$ is the degree of $r$ or $s$.

(1) If $R$ is generated by $x_{1}, \ldots, x_{n}$ and $S$ is generated by $y_{1}, \ldots, y_{m}$ in degree one, then $R \# S$ is generated in degree one by the $x_{i} \# y_{j}$ as $i$ runs from 1 to $n$ and $j$ runs from 1 to $m$.

(2) If the dimension of $R$ is $d$ and the dimension of $S$ is $d^{\prime}$, then the dimension of $R \# S$ is $d+d^{\prime}-1$.

The author would like to thank the referee for pointing out several errors in an earlier version of this paper. 
(3) If $R$ and $S$ are polynomial rings $k\left[x_{1}, \ldots, x_{n}\right]$ and $k\left[y_{1}, \ldots, y_{m}\right]$, where the $x_{i}$ and $y_{j}$ have degree one, then $R \# S$ is a generic determinantal ring with generators corresponding to the $x_{i} \# y_{j}$ divided by the ideal of 2 by 2 minors.

(4) If $k$ is algebraically closed and $R$ and $S$ are normal domains, then $R \# S$ is a normal domain.

The main property of Segre products that we use in this paper is the description of their local cohomology, a formula due to Goto and Watanabe [1]. We denote the homogeneous maximal ideal of $R$ by $\mathfrak{m}_{R}$, that of $S$ by $\mathfrak{m}_{S}$, and that of $R \# S$ by $\mathfrak{m}_{R \# S}$.

Theorem 1. Let $R$ and $S$ be graded rings, and assume that the local cohomologies $H_{m_{R}}^{0}(R)$ and $H_{\mathfrak{m}_{R}}^{1}(R)$ are zero and similarly for $S$. Then for each $q$ we have

$$
H_{\mathfrak{m}_{R \# S}}^{q}(R \# S) \cong R \# H_{\mathfrak{m}_{S}}^{q}(S) \oplus H_{\mathfrak{m}_{R}}^{q}(R) \# S \oplus\left[\oplus_{i+j=q+1} H_{\mathfrak{m}_{R}}^{i}(R) \# H_{\mathfrak{m}_{S}}^{j}(S)\right] .
$$

To illustrate this formula, we consider the case in which $R$ and $S$ are Cohen-Macaulay. Let $d$ be the dimension of $R$ and let $d^{\prime}$ be the dimension of $S$. The Cohen-Macaulay hypothesis means that $H_{\mathfrak{m}_{R}}^{i}(R)=0$ for $i \neq d$ and $H_{\mathfrak{m}_{S}}^{j}(S)=0$ for $j \neq d^{\prime}$. We can see from the above formula that there are three possible nonzero contributions to the local cohomology of $R \# S$, namely $R \# H_{\mathfrak{m}_{S}}^{d^{\prime}}(S)$ in degree $d^{\prime}$, $H_{\mathfrak{m}_{R}}^{d}(R) \# S$ in degree $d$, and $H_{\mathfrak{m}_{R}}^{d}(R) \# H_{\mathfrak{m}_{S}}^{d^{\prime}}(S)$ in degree $d+d^{\prime}-1$. The third of these will definitely not be zero; note that $d+d^{\prime}-1$ is the dimension of $R \# S$. If we assume that $d$ and $d^{\prime}$ are at least 2 (which must be true if the above formula is to hold), then $R \# S$ is Cohen-Macaulay if and only if both of the other summands vanish. Assume that $R_{i} \neq 0$ and $S_{i} \neq 0$ for all $i \geq 0$ (which is true, for example, if $R$ and $S$ are standard graded). For $R \# H_{\mathfrak{m}_{S}}^{d^{\prime}}(S)$ to vanish, for example, it is necessary and sufficient that $H_{\mathfrak{m}_{S}}^{d^{\prime}}(S)$ have no nonzero elements of nonnegative degree. Thus if we wish to construct a normal non-Cohen-Macaulay domain, it suffices to find a normal graded domain with an element of local cohomology of nonnegative degree. The simplest example of this is a polynomial ring in three variables divided by a homogeneous cubic, say $k[x, y, z] /\left(x^{3}+y^{3}+z^{3}\right)$, where $k$ is a field of characteristic not equal to three. Then if $k[a, b]$ is a polynomial ring in two variables, $k[a, b] \# k[x, y, z] /\left(x^{3}+y^{3}+z^{3}\right)$ is a normal non-Cohen-Macaulay domain of dimension 3.

The Segre product is defined for graded rings over a field; however, in our applications we will want to use rings of mixed characteristic. We will use two generalizations of Segre products to the mixed characteristic case. First, we will consider graded rings over an unramified discrete valuation ring $V$ of mixed characteristic, and we will assume that all such rings are torsion-free as $V$-modules. We define the Segre product of two such rings in the same way as we do over a field. The Segre product will then also be torsion-free as a $V$-module. If $R$ and $S$ are such graded rings over $V$, and if $x$ and $y$ are homogeneous elements of the same degree of $R$ and $S$, we will also consider the $\operatorname{ring} R \# S /(p-x \# y)$, where $p$ is the characteristic of the residue field of $V$. Both of these types of rings will be called rings of Segre product type.

As mentioned above, many of the examples of normal non-Cohen-Macaulay rings turn out to be rings of Segre product type. We give three examples; we note also that one of the most commonly used examples in the subject, the ring $k[x, y, z, w] /(x w-y z) \cong k[a, b] \# k[c, d]$, is a Segre product, and the ring $\mathbb{Z}_{p}[y, z, w] /(p w-y z)$, where $\mathbb{Z}_{p}$ is the localization of $\mathbb{Z}$ at the prime ideal $p \mathbb{Z}$, is of Segre product type.

The first example is the mixed characteristic version of the ring $k[a, b] \# k[x, y, z] /\left(x^{3}+y^{3}+z^{3}\right)$ mentioned above. If we take $\mathbb{Z}_{p}[a, b] \# \mathbb{Z}_{p}[x, y, z] /\left(x^{3}+y^{3}+z^{3}\right)$ for some $p \geq 5$ and divide by the ideal generated by $p-a \# x$, we obtain the example worked out in detail at the end of Roberts [8].

The next example is a family of non-Cohen-Macaulay rings discussed in Roberts [7]. The example was the extension of $k[x, y, z, w] /(x w-y z)$ obtained by adjoining a square root of $\prod_{i=0}^{2 n}\left(x-\alpha_{i} z\right)$ for distinct $\alpha_{i} \in k$ for some $n \geq 2$ and taking the integral closure. This integral closure is the Segre product of $k[a, b]$ with $k[u, v, t] /\left(t^{2}-\prod_{i=0}^{2 n}\left(u-\alpha_{i} v\right)\right)$, where $u$ and $v$ have degree 1 , and where $t$ has degree $n$.

The final example was shown to me by Ray Heitmann. It is obtained from the Rees ring of the ideal $(3, a, z)$ in $R=\mathbb{Z}_{3}[a, z] /\left(81+a^{4}+z^{2}\right)$; the integral closure of this Rees ring is the subring $R\left[3 T, a T, z T, z T^{2}\right]$ of $R[T]$. Again it is a ring of Segre product type, obtained from the Segre product 
of $\mathbb{Z}_{3}[x, y]$ and $\mathbb{Z}_{3}[u, a, z] /\left(u^{4}+a^{4}+z^{2}\right)$, where all variables have degree 1 except $z$, which has degree 2 , by dividing by the ideal generated by $3-x \# u$.

\section{A method of Almost Killing LOCAL COHOMOlOGY}

In this section we describe what it means to almost kill local cohomology and present a construction that we will use later to do this for rings of Segre product type. We first recall some definitions.

Let $K$ be a field. By a valuation on $K$ we mean a function $v$ from $K$ to the ordered additive group of rational numbers together with a symbol $\infty$ such that

(1) $v(a b)=v(a)+v(b)$ for all $a$ and $b$ in $K$.

(2) $v(a+b) \geq \min (v(a), v(b))$ for all $a$ and $b$ in $K$.

(3) $v(a)=\infty$ if and only if $a=0$.

In our applications we will consider valuations on integral extensions of local rings with unique maximal ideals, and the valuations will be assumed to take nonnegative values on the ring and positive values on the maximal ideal.

Definition 1. Let $R$ be a ring with a valuation $v$. An $R$-module $M$ is almost zero if for every $m \in m$ and every real number $\epsilon>0$, there is an $\alpha$ in $R$ with $v(\alpha)<\epsilon$ and $\alpha m=0$.

If $R$ is a local or graded ring of dimension $d$ with system of parameters $x_{1}, \ldots, x_{d}$ and $A$ is an $R$ algebra with a valuation, we will say that $A$ is an almost Cohen-Macaulay algebra if the local cohomology $H_{\left(x_{1}, \ldots, x_{d}\right)}^{i}(A)$ is almost zero for $i=0, \ldots, d-1$, and if $A /\left(x_{1}, \ldots x_{d}\right) A$ is not almost zero. We note that the definition is based on a system of parameters of $R$ rather than $A$ and does not say anything about whether $A$ is Cohen-Macaulay or almost Cohen-Macaulay as a ring.

In later sections we will discuss the question of whether the ring $R^{+}$is an almost Cohen-Macaulay $R$-algebra, where $R$ is a local domain and $R^{+}$is its absolute integral closure, the integral closure of $R$ in the algebraic closure of its quotient field. In fact, what we will show is that in certain situations an element $u$ with $u x_{i} \in\left(x_{1}, \ldots, x_{i-1}\right)$ can be almost annihilated in a given integral extension of $R$. If this can be done for all $i=1, \ldots d$, then a standard argument (see Matsumura [6]) shows that the Koszul complex on $x_{1}, \ldots, x_{d}$ is almost exact and thus the local cohomology is almost zero.

3.1. The basic construction. Let $R$ be a normal domain of mixed characteristic $p$ and dimension $d$ over a complete unramified discrete valuation ring $V$ with perfect residue field $k$. Let $p=x_{1}, x_{2}, \ldots, x_{d}$ be a system of parameters for $R$. Let $u$ be an element of $R$ with $u x_{i} \in\left(x_{1}, \ldots, x_{i-1}\right)$ for some $i \geq 3$. In this section we outline a procedure for constructing an extension $C$ of $R$ in which, in the cases we work out below, the image of $u$ modulo $\left(x_{1}, \ldots, x_{i-1}\right)$ is almost zero.

The extension $C$ is constructed as follows. We first adjoin $p^{n}$ th roots of a set of generators of $R$; more precisely, we take a polynomial ring $S=V\left[y_{2}, \ldots, y_{t}\right]$ that maps onto $R$, where $y_{2}, \ldots, y_{d}$ map to $x_{2}, \ldots, x_{d}$. We denote the images of $y_{i}$ by $x_{i}$ for all $i$. We then adjoin a $p^{n}$ th root of $x_{i}$ for all $i$ and all $n$; we do this in a compatible way, so that the $p$ th power of the $p^{n}$ th root we choose is the $p^{n-1}$ st root. Let $T$ denote this ring. We let $C$ denote the ring

$$
C=\left\{a \in T[1 / p] \mid a^{p^{n}} \in T \text { for some } n\right\} .
$$

The question we are studying is whether an element of local cohomology can be almost killed in $C$. We first show how to construct relations in $C$.

We recall some facts about the Fontaine ring and its ring of Witt vectors. This construction is described in more detail in Roberts [8]. We will describe this construction for the ring $T$; the same construction works for $C$ (and in fact for any ring of mixed characteristic).

The Fontaine ring of $T$, denoted $E(T)$, is the projective limit of a system of copies of $T / p T$ indexed over the nonnegative integers, where the maps are the Frobenius map. An element of $E(T)$ is given by a sequence $\left(t_{0}, t_{1}, \ldots,\right)$, where we have $t_{i}^{p} \equiv t_{i-1}$ modulo $p$ for all $i \geq 1$. In particular, for each $i$ the system of $p^{n}$ th roots of $x_{i}$ defines an element of $E(T)$ which we denote $X_{i}$; when $i=1$ we have $x_{1}=p$, and we sometimes denote $X_{1}$ by $P$. Since the residue field $k$ of $V$ is perfect and $k$ is a field contained in $T / p T, k$ is embedded in $E(T)$. We can now define a map $\phi$ from $k\left[y_{2}, \ldots, y_{t}\right]$ to $E(T)$ mapping $y_{i}$ to $X_{i}$. Let $E_{0}$ be image of $\phi$. 
There is a natural map from $E(T)$ to $E(C)$ by functoriality; we will also use the notation $X_{i}$ to denote the corresponding elements of $E(C)$ and $\phi$ to denote the corresponding map from $k\left[y_{2}, \ldots, y_{t}\right]$ to $E(C)$.

In the second part of this construction we take the rings of Witt vectors on $E(T)$ and $E(C)$, which we denote $W(E(T))$ and $W(E(C))$. Since $k$ is contained in $E(T)$ and $E(C)$, the ring of Witt vectors on $k$, which is isomorphic to $V$, is contained in $W(E(T))$ and $W(E(C))$. We now take the Teichmüller elements corresponding to the elements $X_{i}$, that is, the Witt vectors $\left(X_{i}, 0,0, \ldots\right)$; we denote these elements $\left[X_{i}\right]$. We now have maps, which we denote $\psi$, from $S$ to $W(E(T))$ and $W(E(C))$ which send $V$ to $V$ and the $y_{i}$ to $\left[X_{i}\right]$. There are also maps from $W(E(T))$ and $W(E(C))$ to the $p$-adic completions $\hat{T}$ and $\hat{C}$ of $T$ and $C$ respectively; these maps, which we denote $\sigma$, send $[P]$ to $p$, and, more generally, $\left[X_{i}\right]$ to $x_{i}$. We let $W_{0}$ be the image $\psi(S)$ of $S$ in $W(E(T)$ ). Then the above map to $\hat{T}$ induces a surjection from $W_{0}$ onto $R$.

The main property of $C$ is that it behaves well with respect to these constructions in the sense that it satisfies the following two properties (see Roberts [9]).

(1) The map $\sigma$ induces an isomorphism $E(C) / P E(C) \rightarrow C / p C$.

(2) The map $\sigma$ induces an isomorphism $W(E(C)) /(P-p) W(E(C)) \rightarrow \hat{C}$.

It is the second of these properties that allows us to define nontrivial and useful relations on $\hat{C}$. Let $f$ be an element of the kernel of the map from $S$ to $R$. Then the image $\psi(f)$ of $f$ in $W_{0}$ is in the kernel of the map $\sigma$ to $\hat{R}$ and therefore also the composite to $\hat{C}$. By Property 2 above, this implies that $\psi(f)$ is in $(P-p) W(E(C))$. Hence if we compute $\psi(f) /(P-p)$ as a Witt vector, its components will be in $E(C)$.

The next two lemmas give us information about the components of the Witt vector $\psi(f) /(P-p)$ from information about $f$.

Lemma 1. Let $R$ be a graded ring generated by homogeneous elements $x_{i}$, and let $S=V\left[y_{1}, \ldots, y_{t}\right]$ be a polynomial ring mapping onto $R$ as above, where $S$ is graded and $y_{i}$ has the same degree as $x_{i}$ for each i. Let $f\left(y_{i}\right)$ be a homogeneous polynomial in $S$ of degree $k$. Let $\left[X_{i}\right]$ denote the element $\left(X_{i}, 0,0, \ldots\right)$ of $W(E(T))$ for each $i$, and let $f\left(\left[X_{i}\right]\right)=\left(a_{0}, a_{1}, \ldots\right)$ in $W(E(T))$. Then, if we give $X_{i}$ the degree of $x_{i}$ for each $i$, then $a_{j}$ is homogeneous of degree $k p^{j}$ for each $i$.

Proof. We first prove the result for a polynomial with one term; that is, the product of an element of $V$ with a monomial in the $x_{i}$. The corresponding element in $W(E(T))$ is the element of $V$ as a Witt vector times the same monomial in the $\left[X_{i}\right]$ since the Teichmüller map is multiplicative. Let $m$ be this monomial and let $\left(k_{0}, k_{1}, k_{2}, \ldots\right)$ be its coefficient as an element of $W(k)$. Then the product is $\left(k_{0} m, k_{1} m^{p}, k_{2} m^{p^{2}}, \ldots\right)$. Since $m$ has degree $k$ and $k_{i}$ has degree $0, k_{i} m^{p^{i}}$ has degree $k p^{i}$ as required.

The general case will follow from the fact that the sum of two elements $\left(a_{0}, a_{1}, \ldots\right)$ and $\left(b_{0}, b_{1}, \ldots\right)$ for which $a_{i}$ and $b_{i}$ have degree $k p^{i}$ for each $i$ also has this property. Let $\left(s_{0}, s_{1}, \ldots\right)$ be the sum. For $i=0, s_{0}=a_{0}+b_{0}$, so the result is true in this case. Assume now that $s_{i}$ has degree $k p^{i}$ for all $i<j$. Then $s_{j}$ is found from the equation

$$
s_{0}^{p^{j}}+p s_{1}^{p^{j-1}}+\cdots+p^{j} s_{j}=a_{0}^{p^{j}}+p a_{1}^{p^{j-1}}+\cdots+p^{j} a_{j}+b_{0}^{p^{j}}+p b_{1}^{p^{j-1}}+\cdots+p^{j} b_{j} .
$$

The hypothesis implies that each of the terms involving $a_{i}$ or $b_{i}$ has degree $k p^{j}$, and induction implies the same for the terms involving $s_{i}$ for $i<j$. Hence $s_{j}$ has degree $k p^{j}$.

The next Lemma gives the properties we will need of the quotient when divided by $P-p$.

Lemma 2. Let $A$ be a graded subring of $E(T)$ as in the previous lemma, and let $\left(a_{0}, a_{1}, \ldots\right)$ be an element of $W(E(T)))$ such that $a_{i}$ is a homogeneous elements of $A$ of degree $k p^{i}$ for each $i$. Let $\left(a_{0}, a_{1}, \ldots\right)=(P-p)\left(z_{0}, z_{1}, \ldots\right)$ in $E(C)$. Then for each $i \geq 0$ we can write

$$
z_{i}=\frac{a_{0}^{p^{i}}+\sum P^{n_{i j}} b_{i j}}{P^{(i+1) p^{i}}}
$$

where each $n_{i j}$ is a positive integer and $b_{i j}$ is a homogeneous element of $A$ of degree $k p^{i}$. 
Proof. Again we prove this by induction on $i$. We can write the equation defining the $z_{i}$ as

$$
\left(a_{0}, a_{1}, a_{2} \ldots\right)=\left(P z_{0}, P^{p} z_{1}, P^{p^{2}} z_{2}, \ldots,\right)-\left(0, z_{0}^{p}, z_{1}^{p}, \ldots\right) .
$$

Thus for $i=0$ we have $z_{0}=a_{0} / P$, and since $P=P^{(0+1) p^{0}}$, this is in the correct form (here all the other terms are zero).

We now assume that the result holds for $j<i$ and prove that it holds for $i$. The defining equation for $z_{i}$ is

$$
\begin{gathered}
a_{0}^{p^{i}}+p a_{1}^{p^{i-1}}+\cdots+p^{j} a_{j}^{p^{i-j}}+\cdots+p^{i} a_{i}=\left(P z_{0}\right)^{p^{i}}+p\left(P^{p} z_{1}\right)^{p^{i-1}}+\cdots+p^{j}\left(P^{p^{j}} z_{j}\right)^{p^{i-j}}+\cdots+p^{i}\left(P^{p^{i}} z_{i}\right) \\
-p\left(z_{0}^{p}\right)^{p^{i-1}}-\cdots-p^{j}\left(z_{j-1}^{p}\right)^{p^{i-j}}-\cdots-p^{i}\left(z_{i-1}^{p}\right) .
\end{gathered}
$$

Hence, if we solve this equation for $z_{i}$, we conclude that $z_{i}$ is a combination of the other terms in the above expression divided by $p^{i} P^{p^{i}}$. The factor $p^{i}$ will divide the other terms in this expression after the formulas for the $z_{j}$ for $j<i$ are substituted from the general theory of Witt vectors; the factor we have to consider is $P^{p^{i}}$. Thus to complete the proof we must show that each term in the above equation other than $p^{i}\left(P^{p^{i}} z_{i}\right)$ is a sum of terms that can be written in the form $P^{n} a / P^{i p^{i}}$ with $a$ homogeneous of degree $k p^{i}$ and that the only term for which $n=0$ is $a_{0}^{p^{i}}$.

Each of the terms $p^{j} a_{j}^{p^{i-j}}$ is homogeneous of degree $k p^{i}$ and we can take $n=i p^{i}$, so these terms clearly satisfy the required condition.

We next consider an element of the form $p^{j}\left(P^{p^{j}} z_{j}\right)^{p^{i-j}}$. By induction, $z_{j}$ is a sum of terms $P^{k_{m}} b_{m}$ divided by $P^{(j+1) p^{j}}$ with $b_{m}$ homogeneous of degree $k p^{j}$ and exactly one $k_{m}=0$, for which $b_{m}=a_{0}^{p^{j}}$. When this sum is multiplied by $P^{p^{j}}$ and raised to the $p^{i-j}$ th power we obtain a sum of integer multiples of terms of the form

$$
\frac{\left(P^{p^{j}}\right)^{p^{i-j}} \prod_{n} P^{r_{n} k_{m_{n}}} b_{m_{n}}^{r_{n}}}{\left(P^{(j+1) p^{j}}\right)^{p^{i-j}}}
$$

In this product the sum of the $r_{n}$ is $p^{i-j}$, the $k_{m_{n}}$ are positive except for one term (coming from $a_{0}^{p^{j}}$ ) which we compute below, and the $b_{m_{n}}$ are homogeneous of degree $k p^{j}$. It follows that the product of the $b_{m_{n}}^{r_{n}}$ is homogeneous of degree $\left(\sum r_{n}\right) k p^{j}=p^{i-j} k p^{j}=k p^{i}$. Denoting this product $b$, and letting $k=\sum r_{n} k_{m_{n}}$, we can write this term in the form

$$
\frac{P^{p^{i}+k} b}{P^{(j+1) p^{i}}}
$$

The denominator of this term is $\left(P^{(j+1) p^{j}}\right)^{p^{i-j}}=P^{(j+1) p^{i}}$. Since $j \leq i-1$, the highest possible power of $P$ in the denominator is $P^{(i-1+1) p^{i}}=P^{i p^{i}}$. Hence these terms are in the correct form $P^{n} a / P^{i p^{i}}$, and since the numerator has a factor of $P^{i}$, the power $n$ of $P$ in the numerator is positive.

Finally, we consider the terms $p^{j}\left(z_{j-1}^{p}\right)^{p^{i-j}}$. Here we must look at $\left(z_{j-1}^{p}\right)^{p^{i-j}}=z_{j-1}^{p^{i-j+1}}$ for $1 \leq j \leq i$. Following the same computation as above, we see by applying the induction hypothesis to $z_{j-1}$ that $z_{j-1}^{p^{i-j+1}}$ can be written as a sum of terms of the form $b P^{k}$ over a power of $P$, where $b$ is homogeneous of degree $k p^{i}$. We next determine the possible power of $P$ in the denominator. Since the denominator for $z_{j-1}$ is $P^{j p^{j-1}}$, the denominator for $z_{j-1}^{p^{i-j+1}}$ will be $\left(P^{j p^{j-1}}\right)^{p^{i-j+1}}=P^{j p^{i}}$. When $j<i$, the power of $P$ in the denominator will be less than $i p^{i-1}$, and all the terms can be written in the form $P^{n} a / P^{i p^{i}}$ with $n$ positive. When $j=i$ (the term $p^{i} z_{i-1}^{p}$ ), the denominator is $P^{i p^{i}}$, and by induction the only term in the expression for $z_{i-1}$ which does not have a factor of $P$ is $a_{0}^{p^{i-1}}$. Hence when $z_{i-1}$ is raised to the $p$ th power, the only term without a factor of $p$ will be $\left(a_{0}^{p^{i-1}}\right)^{p}=a_{0}^{p^{i}}$.

Hence $z_{i}$ has the stated form, so this completes the proof.

A similar but simpler argument shows the following.

Proposition 1. If in the situation of the above lemmas the element $f$ in the kernel of the map from $S$ to $R$ is divisible by $x_{j}$, then $z_{i}$ is divisible by $X_{j}^{p^{i}}$. 


\section{A general SUfFicient CONDition FOR the EXISTENCE OF SMALl ANNiHilators.}

In this section we outline a method for showing that a relation of the form $u x_{i} \in\left(x_{1}, \ldots, x_{i-1}\right)$ can be almost killed in $C$ and a sufficient condition that implies that this method will work.

Lemma 2 gives the form of certain elements in $E(C)$ that are derived from elements of the kernel of the original map from $S$ to $R$ that presented $R$ as the homomorphic image of a polynomial ring. It was assumed that the elements of the kernel were homogeneous; if this is not the case, the elements of $E(C)$ derived from $\left(a_{0}, a_{1}, \ldots,\right) /(P-p)$ still have the form

$$
z_{i}=\frac{a_{0}^{p^{i}}+\sum P^{n_{i j}} b_{i j}}{P^{(i+1) p^{i}}}
$$

where each $n_{i j}$ is a positive integer and each $b_{i j}$ is a polynomial in the $a_{i}$.

If $u$ is an element of $R$ satisfying a relation $u x_{i} \in\left(x_{1}, \ldots, x_{i-1}\right)$, we wish to find a small element $\gamma$ such that $\gamma u \in\left(x_{1}, \ldots, x_{i-1}\right) C$. We are assuming that $x_{1}=p$, so this means that we want to find $\gamma$ with $\gamma u \in\left(x_{2}, \ldots, x_{i-1}\right)(C / p C)$. Since $C / p C \cong E(C) / P E(C)$, it suffices to find such a $\gamma$ in $E(C)$ with $\gamma \bar{u} \in\left(X_{2}, \ldots, X_{i-1}\right) E(C) / P E(C)$, where $\bar{u}$ is a lifting of $u$. We first show how to find relations in $E(C) / P E(C)$ coming from Lemma 2 .

Let $m=p^{n}-1$ for some $n>0$. Then in the formula for $z_{m}$ the denominator is

$$
P^{(m+1) p^{m}}=P^{\left(p^{n}\right) p^{m}}=P^{p^{n+m}} .
$$

We now take the $p^{n+m}$ th root of $z_{m}$ in $E(C)$ (which is perfect). The denominator is now $P$. Hence if we multiply this element by $P$, the result is zero in $E(C) / P E(C)$. This relation in $E(C) / P E(C)$ will be in the form

$$
a_{0}^{1 / p^{n}}+\sum_{j>0} h_{j n} P^{j / p^{n+m}}=0
$$

for some coefficients $h_{j n}$. This discussion holds whether the ring is graded or not; however, if the original element in $S$ is homogeneous of degree $k$, then Lemma 2 implies that $a_{0}^{1 / p^{n}}$ and the $h_{j n}$ are homogeneous of degree $k / p^{n}$.

We now assume that there is a function $\mu$ from $E(C) / P E(C)$ to an ordered abelian group together with an element $\infty$ satisfying conditions that we will outline below. An example to keep in mind is the grading in Lemma 2; in our examples we will need a more general function, but it is reasonable to think of it as a generalization of a grading on a ring. We assume that the function $\mu$ satisfies $\mu(x y) \geq \mu(x)+\mu(y)$ and $\mu(x+y) \geq \min (\mu(x), \mu(y))$. The main assumption is that there is a set of elements in $S$ that map to zero in $R$ such that the associated relations in $E(C) / P E(C)$ have very nice properties. Let $u$ be an element of $R$ such that $u x_{i} \in\left(x_{1}, \ldots, x_{i-1}\right)$.

Let $f_{1}, \ldots, f_{t}$ be a set of elements in the kernel of the map from $S$ to $R$. For each $f_{\ell}$, and for each $n \geq 0$, let $\sum_{j \geq 0} h_{\ell j n} P^{j / p^{m}}=0$ be the corresponding relation in $E(C) / P E(C)$ constructed as above. The condition we need is that any element $v$ with $\mu(v)>\mu(u)$ can be expressed as an element of $\left(X_{1}, \ldots, X_{i-1}\right)$ modulo the leading terms of these elements, that is, the elements $h_{\ell 0 n}$, for large enough $n$. To make this work we need a slightly more precise condition. For an integer $n$, let $\bar{C}_{n}$ denote the quotient $E(C) / P^{1 / p^{n+m}} E(C)$, and let $I_{n}$ denote the ideal of $\bar{C}_{n}$ generated by the leading terms $h_{10 n}, \ldots, h_{t 0 n}$.

Assume that the following conditions hold for the function $\mu$ and for some integer $s$.

(1) We have $\mu(\bar{u}) \geq s$.

(2) For all $\delta>0$, there is an integer $n$ such that if $\mu(v) \geq s+\delta$, then $v$ is in the ideal generated by $X_{1}, \ldots, X_{i-1}$ in $\bar{C}_{n} / I_{n}$.

(3) Condition 2 says that we can write

$$
v \equiv w+\sum_{i=1}^{t} e_{i}\left(\sum_{j \geq 0} h_{i j n} P^{j / p^{n+m}}\right) \text { modulo } I_{n}
$$


for some $w \in\left(X_{1}, \ldots, X_{i-1}\right)$ and $e_{i} \in E(C)$. We require that we can choose $w$ and the $e_{i}$ so that if we write

$$
v-w-\sum_{i=1}^{t} e_{i}\left(\sum_{j \geq 0} h_{i j n} P^{j / p^{n+m}}\right)=\sum_{j>0} c_{j} P^{j / p^{n+m}},
$$

then $c_{j}$ satisfies $\mu\left(c_{j}\right) \geq s+\delta$ for all $j$.

Suppose that this holds. We claim that then there is a small $\gamma$ with $\gamma \bar{u} \in\left(X_{1}, \ldots, X_{i-1}\right) E(C)$.

To see this, we take $\epsilon>0$, and let $v$ be a valuation on $R$ extended to $T$ and $C$. Choose an element $r$ with $\mu(r)>0$ and $v(r)>0$; by replacing $r$ by a suitable $p$ th root modulo $p$ we may assume that $v(r)<\epsilon$. Let $\delta=\mu(r)$ and choose $n$ as in the second condition above. By that condition we can find elements $g_{1}, \ldots, g_{t}$ and $g$ in $E(C)$ such that

$$
r \bar{u}=w+\sum g_{\ell} h_{\ell 0 n}
$$

modulo the ideal generated by $P^{1 / p^{n+m}}$, where $w \in\left(X_{1}, \ldots, X_{i-1}\right)$. By the third condition, the $g_{\ell}$ can be chosen so that if we write

$$
r \bar{u}-w-\sum g_{\ell} h_{\ell 0 k}=\sum_{j>0} c_{j} P^{j / p^{n+m}},
$$

we have that $\mu\left(c_{j}\right) \geq s+\delta$ for all $j$, so we can continue this process.

Assume by induction that for a given integer $\alpha$ we have $g_{\ell \alpha}$ such that

$$
r \bar{u} \equiv w+\sum_{\ell=1}^{t} g_{\ell \alpha}\left(\sum_{j \geq 0} h_{\ell j n} P^{j / p^{n+m}}\right)
$$

modulo $P^{\alpha / n+m}$, where $w \in\left(X_{1}, \ldots, X_{i-1}\right)$ and where if we write

$$
r \bar{u}-w-\sum_{\ell=1}^{t} g_{\ell \alpha}\left(\sum_{j \geq 0} h_{\ell j n} P^{j / p^{n+m}}\right)=\sum_{j \geq \alpha} c_{j} P^{j / p^{n+m}},
$$

the coefficients $c_{j}$ of $P^{j / p^{n+m}}$ satisfy $\mu\left(c_{j}\right) \geq s+\delta$ for all $j \geq \alpha$. In particular, $\mu\left(c_{\alpha}\right) \geq s+\alpha$. Hence we can write

$$
c_{\alpha} \equiv w^{\prime}+\sum g_{\ell}^{\prime} h_{\ell 0 n}
$$

modulo $P^{1 / p^{n+m}}$ with $w^{\prime} \in\left(X_{1}, \ldots, X_{i-1}\right)$ and satisfying the condition that if we write

$$
c_{\alpha}-w^{\prime}-\sum g_{\ell}^{\prime} \sum_{j} h_{\ell j n} P^{j / p^{n+m}}=\sum_{j>0} c_{j}^{\prime} P^{j / p^{n+m}},
$$

the coefficients $c_{j}^{\prime}$ satisfy $\mu\left(c_{j}^{\prime}\right) \geq s+\delta$.

We now substitute this expression for $c_{\alpha}$ in the inductive expression $\left(^{*}\right)$ for $r \bar{u}$ and obtain

$$
\begin{gathered}
r \bar{u}=w+\sum_{\ell=1}^{t} g_{\ell \alpha}\left(\sum_{j \geq 0} h_{\ell j n} P^{j / p^{n+m}}\right)+\sum_{j \geq \alpha} c_{j} P^{j / p^{n+m}}= \\
w+\sum_{\ell=1}^{t} g_{\ell \alpha}\left(\sum_{j \geq 0} h_{\ell j n} P^{j / p^{n+m}}\right)+c_{\alpha} P^{\alpha / p^{n+m}}+\sum_{j>\alpha} c_{j} P^{j / p^{n+m}}= \\
w+\sum_{\ell=1}^{t} g_{\ell \alpha}\left(\sum_{j \geq 0} h_{\ell j n} P^{j / p^{n+m}}\right)+P^{\alpha / p^{n+m}}\left(w^{\prime}+\sum g_{\ell}^{\prime} \sum_{j} h_{\ell j n} P^{j / p^{n+m}}+\sum_{j>0} c_{j}^{\prime} P^{j / p^{n+m}}\right)+\sum_{j>\alpha} c_{j} P^{j / p^{n+m}}
\end{gathered}
$$

If we let $w^{\prime \prime}=w+P^{\alpha / p^{n+m}} w^{\prime}$, let $g_{\ell}^{\prime \prime}=g_{\ell \alpha}+P^{\alpha / p^{n+m}} g_{\ell}^{\prime}$, and let $c_{j}^{\prime \prime}=c_{j}+c_{j-\alpha}^{\prime}$, we have

$$
r \bar{u}=w^{\prime \prime}+\sum_{\ell=1}^{t} g_{\ell}^{\prime \prime}\left(\sum_{j \geq 0} h_{\ell j n} P^{j / p^{n+m}}\right)+\sum_{j \geq \alpha+1} c_{j}^{\prime \prime} P^{j / p^{n+m}} .
$$


Since $w$ and $w^{\prime}$ are in $\left(X_{1}, \ldots, X_{i-1}\right)$ so is $w^{\prime \prime}$, and the condition that $\mu\left(v+v^{\prime}\right) \geq \min \left(\mu(v), \mu\left(v^{\prime}\right)\right)$ implies that $\mu\left(c_{j}^{\prime \prime}\right) \geq s+\delta$ for all $j$. Thus we can continue this process, and when we arrive at $\alpha=p^{n+m}$, we can conclude that $r \bar{u} \in\left(X_{1}, \ldots, X_{i-1}\right)$.

We remark that if the function $u$ is defined by a grading and everything involved is homogeneous, then the third condition will be automatic if we assume that the elements in the expression 2 are also homogeneous of the correct degree.

In the next section we begin to compute how the procedure described in this section can be carried out for rings of Segre product type.

\section{The Segre product of two Cohen-Macaulay Rings.}

Let $R$ and $S$ be graded Cohen-Macaulay rings of dimensions $d^{\prime}$ and $d$ respectively over a field $k$; assume that $d \leq d^{\prime}$. We assume that $d$ and $d^{\prime}$ are at least 2 . Let $a_{1}, \ldots, a_{d^{\prime}}$ be a homogeneous system of parameters for $R$ and let $x_{1}, \ldots, x_{d}$ be a homogeneous system of parameters for $S$. We assume that the $a_{i}$ and the $x_{i}$ all have the same degree.

Proposition 2. A system of parameters for $R \# S$ is

$$
a_{1} x_{1}, a_{2} x_{1}+a_{1} x_{2}, \ldots, a_{d} x_{1}+\cdots+a_{1} x_{d}, \ldots, a_{d^{\prime}-d+1} x_{d}+\cdots+a_{d^{\prime}} x_{1}, \ldots, a_{d^{\prime}} x_{d} .
$$

Since the number of elements in this sequence is $d+d^{\prime}-1$, which is the dimension of $R \# S$, it suffices to show that the quotient after dividing by the ideal generated by these elements has dimension 0 . Since $R$ and $S$ are finite extensions of the polynomial rings $k\left[a_{1}, \ldots, a_{d^{\prime}}\right]$ and $k\left[x_{1}, \ldots, x_{d}\right]$ respectively, we may assume that $R$ and $S$ are these polynomial rings. To show that the quotient has dimension zero, we must show that any prime ideal $\mathfrak{p}$ containing all the elements in the above list contains $a_{i} x_{j}$ for all $i$ and $j$. We prove this by induction on $i+j$. If $i+j=2$, the only element is $a_{1} x_{1}$, which is a generator of the ideal so is certainly in $\mathfrak{p}$. We take $k>2$ and assume that $a_{i} x_{j}$ is in $\mathfrak{p}$ when $i+j<k$. If $i, j$ and $i^{\prime}, j^{\prime}$ are distinct pairs with $i+j=i^{\prime}+j^{\prime}=k$, we have $\left(a_{i} x_{j}\right)\left(a_{i^{\prime}} x_{j^{\prime}}\right)=\left(a_{i} x_{j^{\prime}}\right)\left(a_{i^{\prime}} x_{j}\right)$, and either $i+j^{\prime}$ or $i^{\prime}+j$ is less than $k$, so the second product is in $\mathfrak{p}$ by induction. Hence the product of any two distinct elements $a_{i} x_{j}$ with $i+j=k$ is in $\mathfrak{p}$. Suppose some $a_{r} x_{s}$ with $r+s=k$ is not in $\mathfrak{p}$. Then for any other $i, j$ with $i+j=k$, since $\left(a_{i} x_{j}\right)\left(a_{r} x_{s}\right) \in \mathfrak{p}$ but $\left(a_{r} x_{s}\right) \notin \mathfrak{p}$, we have $\left(a_{i} x_{j}\right) \in \mathfrak{p}$. On the other hand, the sum of $a_{i} x_{j}$ over all $i$ and $j$ with $i+j=k$ is in $\mathfrak{p}$, so $a_{r} x_{s}$ must be in $\mathfrak{p}$ as well. This concludes the proof.

If $R$ and $S$ are Cohen-Macaulay, the local cohomology of their Segre product is relatively simple; it is also this situation, as pointed out above, that provides a major source of examples of non-CohenMacaulay normal domains. Then $R \# S$ has dimension $d+d^{\prime}-1$, and one non-trivial local cohomology module is in degree $d+d^{\prime}-1$. The only other possibilities are in degrees $d$ and $d^{\prime}$. We assume as above that $d \leq d^{\prime}$. We will consider the relations between the elements of the system of parameters in degrees $d$ and $\overline{d^{\prime}}$. First we prove a result on relations in the tensor product $R \otimes_{k} S$.

Proposition 3. Let notation be as above. Assume first that $d<d^{\prime}$. Let

$$
I=\left\{u \in R \otimes_{k} S \mid u\left(a_{d+1} x_{1}+\cdots+a_{2} x_{d}\right) \in\left(a_{1} x_{1}, a_{2} x_{1}+a_{1} x_{2}, \ldots, a_{d} x_{1}+\cdots+a_{1} x_{d}\right)\right\} .
$$

Then

$$
\begin{aligned}
& \text { If } d=d^{\prime} \text {, we let } \\
& \qquad I=\left\{u \in R \otimes_{k} S \mid u\left(a_{d} x_{2}+\cdots+a_{2} x_{d}\right) \in\left(a_{1} x_{1}, a_{2} x_{1}+a_{1} x_{2}, \ldots, a_{d} x_{1}+\cdots+a_{1} x_{d}\right)\right\} .
\end{aligned}
$$

Then

$$
I=\left(a_{1} x_{1}, a_{2} x_{1}+a_{1} x_{2}, \ldots, a_{d} x_{1}+\cdots+a_{1} x_{d}, a_{1}^{d}, x_{1}^{d}\right) .
$$

The proof is by induction on $d$. We first note that since $R$ and $S$ are Cohen-Macaulay, they are flat extensions of the polynomial rings $k\left[a_{1}, \ldots, a_{d^{\prime}}\right]$ and $k\left[x_{1}, \ldots, x_{d}\right]$, so $R \otimes_{k} S$ is a flat extension of $k\left[a_{1}, \ldots, a_{d^{\prime}}\right] \otimes k\left[x_{1}, \ldots, x_{d}\right]$. Hence it suffices to prove the result for the polynomial rings. Let $A$ denote $R \otimes S$. 
We first prove the case $d=2$. If $d^{\prime}=2$, we are considering the sequence

$$
a_{1} x_{1}, a_{2} x_{1}+a_{1} x_{2}, a_{2} x_{2}
$$

and the ideal $I=\left\{u \in A \mid u a_{2} x_{2} \in\left(a_{1} x_{1}, a_{2} x_{1}+a_{1} x_{2}\right)\right\}$.

This is a simple case, but we will work it out in detail since the general argument works the same way. We compute the homology of the Koszul complex on the sequence $a_{1} x_{1}, a_{2} x_{1}+a_{1} x_{2}, a_{2} x_{2}$ in degree one. Let $K_{\bullet}$ denote the Koszul complex on $a_{2} x_{1}+a_{1} x_{2}, a_{2} x_{2}$. Then the Koszul complex we are interested in is the total complex of the double complex obtained by tensoring $K_{\bullet}$ with the complex $0 \rightarrow A \stackrel{a_{1} x_{1}}{\rightarrow} A \rightarrow 0$. This gives the double complex

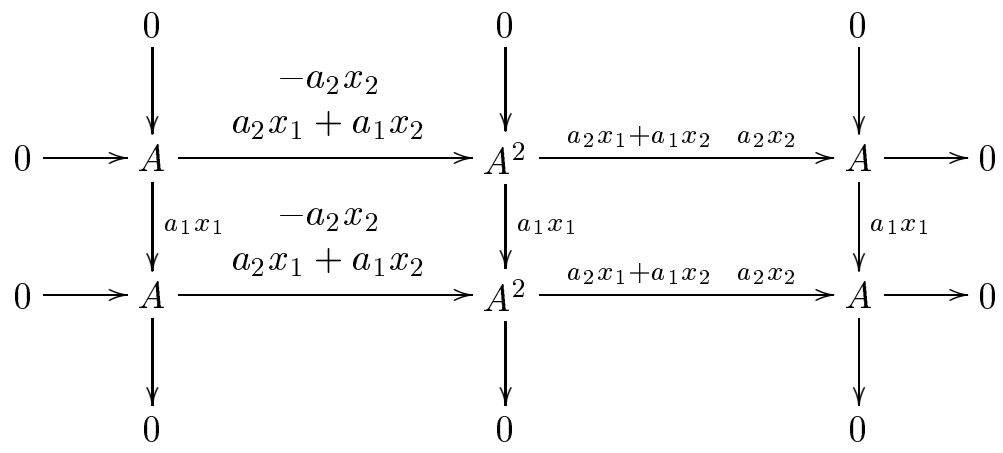

If we take the homology of the columns of this double complex, we obtain the Koszul complex on $a_{2} x_{1}+a_{1} x_{2}, a_{2} x_{2}$ over the ring $A /\left(a_{1} x_{1}\right)$, so it suffices to compute the homology of this complex. By using the short exact sequence

$$
0 \rightarrow A /\left(a_{1}\right) \stackrel{x_{1}}{\rightarrow} A /\left(a_{1} x_{1}\right) \rightarrow A /\left(x_{1}\right) \rightarrow 0,
$$

we further reduce the problem to computing the Koszul complex of $a_{2} x_{1}, a_{2} x_{2}$ on $A /\left(a_{1}\right)$ and of $a_{1} x_{2}, a_{2} x_{2}$ on $A /\left(x_{1}\right)$. The homology of the Koszul complex of $a_{2} x_{1}, a_{2} x_{2}$ on $A /\left(a_{1}\right)$ in degree 1 is generated by $\left(-x_{2}, x_{1}\right)$, and the image of $\left(-x_{2}, x_{1}\right)$ in $\left(A /\left(a_{1} x_{1}\right)\right)^{2}$ is $\left(-x_{1} x_{2}, x_{1}^{2}\right)$. The homology of the Koszul complex of $a_{1} x_{2}, a_{2} x_{2}$ on $A /\left(x_{1}\right)$ in degree 1 is generated by $\left(-a_{2}, a_{1}\right)$, and a simple diagram chase shows that the kernel of the map from this homology to the homology of the Koszul complex of $a_{2} x_{1}, a_{2} x_{2}$ on $A /\left(a_{1}\right)$ in degree zero is generated by $\left(-a_{1} a_{2}, a_{1}^{2}\right)$. Putting these computations together, we see that the ideal $I=\left\{u \in A \mid u a_{2} x_{2} \in\left(a_{1} x_{1}, a_{2} x_{1}+a_{1} x_{2}\right)\right\}$ is generated by $a_{1} x_{1}, a_{2} x_{1}+a_{1} x_{2}, a_{1}^{2}$, and $x_{1}^{2}$ as claimed.

The other cases are proven by the same method of factoring out the complex $0 \rightarrow A \stackrel{a_{1} x_{1}}{\rightarrow} A \rightarrow 0$ and using the short exact sequence $0 \rightarrow A /\left(a_{1}\right) \rightarrow A /\left(a_{1} x_{1}\right) \rightarrow A /\left(x_{1}\right) \rightarrow 0$; in most cases we then apply induction to complete the computation.

We next consider the general case where $d=2$. We assume now that $d^{\prime}>2$.

We have $I=\left\{u \in A \mid u\left(a_{3} x_{1}+a_{2} x_{2}\right) \in\left(a_{1} x_{1}, a_{2} x_{1}+a_{1} x_{2}\right)\right\}$. The computation of $I$ is the same as that where $d^{\prime}=2$ except that the restriction to $A /\left(a_{1}\right)$ is the Koszul complex on $a_{2} x_{1}, a_{3} x_{1}+a_{2} x_{2}$, which is exact in degree one. Hence $I=\left(a_{1} x_{1}, a_{2} x_{1}+a_{1} x_{2}, a_{1}^{2}\right)$.

For $d>2$, we use the same method together with induction on $d$. If $d=d^{\prime}$, we are considering the ideal $I=\left\{u \in R \otimes_{k} S \mid u\left(a_{d} x_{2}+\cdots+a_{2} x_{d}\right) \in\left(a_{1} x_{1}, a_{2} x_{1}+a_{1} x_{2}, \ldots, a_{d} x_{1}+\cdots+a_{1} x_{d}\right)\right\}$. As before, we consider the Koszul complex on $a_{2} x_{1}+a_{1} x_{2}, \ldots, a_{d} x_{1}+\cdots+a_{1} x_{d}, a_{d} x_{2}+\cdots+a_{2} x_{d}$ restricted to $A /\left(a_{1} x_{1}\right)$. The restriction to $A /\left(a_{1}\right)$ gives a similar situation where the dimension in the $a_{i}$ is $d-1$ and that in the $x_{i}$ is $d$, and we are computing the homology in the case where the lower dimension is $d-1$. By induction, this homology is generated by $x_{1}^{d-1}$, and, as in the case $d=d^{\prime}=2$, pushing this element into $A /\left(a_{1} x_{1}\right)$ gives another factor of $x_{1}$, giving $x_{1}^{d}$. The restriction to $A /\left(x_{1}\right)$ is the same with the variables interchanged, giving a generator $a_{1}^{d}$. Hence we have

$$
I=\left(a_{1} x_{1}, a_{2} x_{1}+a_{1} x_{2}, \ldots, a_{d} x_{1}+\cdots+a_{1} x_{d}, a_{1}^{d}, x_{1}^{d}\right) .
$$


If $d^{\prime}>d$, we follow the same steps, the only difference being that the restriction to $A /\left(a_{1}\right)$ is now exact. Hence the only generator is $a_{1}^{d}$, and we have

$$
I=\left(a_{1} x_{1}, a_{2} x_{1}+a_{1} x_{2}, \ldots, a_{d} x_{1}+\cdots+a_{1} x_{d}, a_{1}^{d}\right) .
$$

This proposition describes the relations between the elements in a system of parameters of $R \# S$ in the larger ring $R \otimes S$. The result says that any element in degree $d$, where $d$ is the dimension of $R$, is a multiple of $a_{1}^{d}$. However, to be in the subring $R \# S$ it must be a sum of elements of the form $a_{1}^{d} \# s$; a simple computation shows that any element of this form does give a relation, so the contributions to the local cohomology is generated by elements of this type. The actual homology thus depends on the number of elements $s$ in $S$ of degree equal to $d$ times the degree of $a_{1}$ for which $a_{1}^{d} \# s$ is not in the ideal $\left(a_{1} x_{1}, a_{2} x_{1}+a_{1} x_{2}, \ldots, a_{d} x_{1}+\cdots+a_{1} x_{d}\right)$.

In degree $d^{\prime}$, the situation is more complicated, as there will be relations involving the $a_{i}$ as well as the element $x_{1}^{d^{\prime}}$. We will not pursue this here, but the multiples of $x_{1}^{d^{\prime}}$ give the generators of $H_{\mathfrak{m}_{R \# S}}^{d^{\prime}}(R \# S)$ similar to the situation in degree $d$. Our computations will apply to both of these types of elements.

\section{The Almost VANishing of THE GeneRATORS OF HOMOLOGY}

Let $R$ and $S$ be as above, with $a_{1}, \ldots, a_{d^{\prime}}$ a system of parameters for $R$ and $x_{1}, \ldots, x_{d}$ a system of parameters for $S$. We have shown above that the homology of the Koszul complex on a system of parameters of $R \# S$ in relevant degrees is generated by elements of the form $a_{1}^{d} \# s$ in degree $d$ and $r \# x_{1}^{d^{\prime}}$ in degree $d^{\prime}$, where $r$ and $s$ have degrees $d^{\prime} e$ and $d e$ respectively, where $e$ is the degree of $a_{i}$ or of $x_{j}$. In this section we show that these elements are almost zero when extended to the $\operatorname{ring} C$. We do the case of $a_{1}^{d} \# s$; the other case is similar. We will use $e$ to denote the degrees of the $a_{i}$ and $x_{j}$ as above.

We will show that there is a function $\mu$ as in section 4 with the required properties. We first note that our assumption is that $R \# S$ is of Segre product type, which means that it is a Segre product of torsion-free rings over a discrete valuation ring or such a product divided by an element of the form $p-a_{1} \# x_{1}$. We assume that the first element of a system of parameters is $p$, and when we divide by $p$ we are in the case of a Segre product over a field. Thus the conditions of the previous section apply.

Proposition 4. Let $s \in S$ have degree nde and be a polynomial in the $x_{i}$. Then $\left(a_{1}\right)^{\text {nd }} s$ is in the ideal $\left(\left(a_{1} x_{1}\right)^{n},\left(a_{2} x_{1}\right)^{n}+\left(a_{1} x_{2}\right)^{n} \ldots,\left(a_{d} x_{1}\right)^{n}+\cdots+\left(a_{1} x_{d}\right)^{n}\right)$.

Proof. We can assume that $s$ is a monomial in the $x_{i}$. We will prove the following statement: if $1 \leq j \leq i \leq d$, then if $s$ is a monomial of degree nie with a factor $x_{j}^{n}$, then $a_{1}^{n i} s \in\left(\left(a_{1} x_{1}\right)^{n},\left(a_{2} x_{1}\right)^{n}+\right.$ $\left.\left(a_{1} x_{2}\right)^{n} \ldots,\left(a_{d} x_{1}\right)^{n}+\cdots+\left(a_{1} x_{d}\right)^{n}\right)$.

The proof is by double induction on $i$ and $j$. If $j=1$, then $a_{1}^{n i} s$ has a factor $\left(a_{1} x_{1}\right)^{n}$, so the result is clear. Suppose that $j>1$. Then we can write $a_{1}^{n i} s=\left(a_{1} x_{j}\right)^{n}\left(a_{1}^{n(i-1)} s^{\prime}\right)$ for some $s^{\prime}$ of degree $n(i-1) e$. We can now use the element $\left(a_{j} x_{1}\right)^{n}+\left(a_{j-1} x_{2}\right)^{n}+\cdots+\left(a_{1} x_{j}\right)^{n}$ of the ideal to replace $\left(a_{1} x_{j}\right)^{n}\left(a_{1}^{n(i-1)} s^{\prime}\right)$ by the sum of the terms $-\left(a_{j+1-k} x_{k}\right)^{n}\left(a_{1}^{n(i-1)} s^{\prime}\right)$ for $k=1, \ldots, j-1$. Write $s^{\prime}$ as a product $s_{1} s_{2}$, where $s_{1}$ has degree $n e$ and $s_{2}$ has degree $n(i-2) e$. We can then write

$$
\left(a_{j+1-k} x_{k}\right)^{n}\left(a_{1}^{n(i-1)} s^{\prime}\right)=\left(a_{j+1-k} x_{k}\right)^{n}\left(a_{1}^{n(i-1)} s_{1} s_{2}\right)=\left(a_{1}^{n(i-1)} x_{k}^{n} s_{2}\right)\left(a_{j+1-k}^{n} s_{1}\right) .
$$

By double induction the first factor in the product on the left is in $\left(\left(a_{1} x_{1}\right)^{n},\left(a_{2} x_{1}\right)^{n}+\left(a_{1} x_{2}\right)^{n} \ldots,\left(a_{d} x_{1}\right)^{n}+\right.$ $\left.\cdots+\left(a_{1} x_{d}\right)^{n}\right)$, so this completes the proof.

Extend the set $x_{1}, \ldots, x_{d}$ to a set $x_{1}, \ldots, x_{t}$ of homogeneous generators of $S$ over $V$. Since each of $x_{d+1}, \ldots, x_{t}$ is integral over $V\left[x_{1}, \ldots, x_{d}\right]$, there exists a constant $b$ such that if $s$ is a monomial in the $x_{i}$ for all $i$ (not just up to $d$ ) of degree $n+b$, then it is a linear combination of monomials that have a factor which is a monomial in $x_{1}, \ldots x_{d}$ of degree $n$ (where the degree is in the graded ring $\mathrm{S}$ ). In fact, we can reduce the monomial to a combination of monomials in which the power of each $x_{i}$ for $i>d$ is bounded by a fixed bound, and the sum of these bounds times the degrees of the corresponding $x_{i}$ gives a value of $b$ that will work.

We now define the function $\mu$ and the relations as in section 4 . The function $\mu$ is defined on $E(C) / P E(C)$; we use the fact that $E(C) / P E(C) \cong C / p C$ and define $\mu$ on $C / p C$. We let $\mu(v)=\infty$ for 
$v \in p C$. For general $v$ we wish to let $\mu(v)$ be the supremum of rational numbers $r$ such that $a_{1}^{r}$ divides $v$. The problem is that $a_{1}$ is an element of $R$ not of $R \# S$, so we have to make a more complicated definition. We note that the extension from $R$ to the $\operatorname{ring}$ we denoted $T$ was obtained by adjoining the $p^{n}$ th roots of generators of $R \# S$; we denote an element of $T$ which can be represented as a homogeneous polynomial $r$ in the $p^{n}$ th roots of the $a_{i}$ times a homogeneous polynomial $s$ in the $p^{n}$ th roots of the $x_{i}$ for some $n$ as $r \# s$, extending the notation on $R \# S$. We then define $\mu(v)$ to be the supremum of rational numbers $q$ so that $v$ can be written as a sum of elements $c_{i}$ such that each $c_{i}$ is a multiple of some $r \# s$ where $r$ is divisible by $a_{1}^{q}$. It is clear that $\mu\left(v v^{\prime}\right) \geq \mu(v)+\mu\left(v^{\prime}\right)$ and $\mu\left(v+v^{\prime}\right) \geq \min \left(\mu(v), \mu\left(v^{\prime}\right)\right)$. We now have to check the three conditions of section 4 .

First of all, if we let $s=d$, then $\mu(u)=\mu\left(a_{1}^{d} \# s\right) \geq d=s$.

We next define the functions $f_{1}, \ldots, f_{t}$. Let $e$ be the degree of $a_{1}$ as above, and let the elements $x_{1}, \ldots, x_{d}, x_{d+1}, \ldots, x_{d+t}$ be generators for $S$. For each $\ell$ between 1 and $t$ let $g_{\ell}$ be a homogeneous monic polynomial in $x_{d+\ell}$ of degree $m_{\ell} e$ for some $m_{\ell}$ with coefficients in $k\left[x_{1}, \ldots, x_{d}\right]$. We then let $f_{\ell}=a_{1}^{m_{\ell}} \# g_{\ell}$.

Since the functions $f_{t}$ we just defined and the notation are not quite the same as those in the general procedure described in earlier sections, we will briefly outline the differences. In the general description we had a ring $R$ and a polynomial ring mapping onto it; the images of the variables we called generators of $R$. Here the ring corresponding to $R$ is $R \# S$, and we have not given a complete set of generators for $R \# S$. Of course, a complete set of generators for $R \# S$ can be written in terms of generators for $R$ and $S$, but their form will depend on the degrees of the generators of $R$ and $S$. It is admittedly somewhat of an abuse of notation, but we will consider the elements $a_{1}^{m_{\ell}} \# g_{\ell}$ as polynomials in the generator $a_{1}$ for $R$ and the generators $x_{i}$ of $S$ which map to zero in $R \# S$.

Let $\delta>0$. We need to find an integer $k$ such that if $v$ is an element of $C / p C$ with $\mu(v) \geq d+\delta$, then $v$ is in the ideal generated by $a_{1} x_{1}, a_{2} x_{1}+a_{1} x_{2}, \ldots, a_{d} x_{1}+\cdots+a_{1} x_{d}$ modulo the $p^{k}$ th roots of the $f_{\ell 0}$. We may assume that $v=a_{1}^{\beta} r \# s$, where $\beta$ is a rational number greater than $d+\delta$ with denominator a power of $p$ and $s$ is a homogeneous element which is a polynomial in the $x_{j}$ with rational exponents. If we look modulo the polynomials $g_{\ell}$, as mentioned above, there is an integer $b$ such that any monomial in the $x_{j}$ of degree $n+b$ is congruent to a linear combination of polynomials each of which has a factor of degree at least $n$ in $x_{1}, \ldots, x_{d}$. If we take $p^{k}$ th roots, we may conclude that a polynomial of degree $(e d+b) / p^{k}$ is congruent to a linear combination of polynomials with a factor of degree $e d$ in the $p^{k}$ th roots of $x_{1}, \ldots, x_{d}$ modulo the ideal generated by the polynomials $g_{\ell k}$, where $g_{\ell k}$ is the polynomial obtained from $g_{\ell}$ by taking $p^{k}$ th roots of the terms. Hence if we choose $n$ such that $b / p^{n}<\delta$ and assume that $\mu\left(a_{1}^{\beta} r \# s\right) \geq d+\delta$, which implies that the degree of $s$ is at least $e d+b$, we can reduce $s$ to a finite linear combination of elements modulo $I_{n}$ which has a factor which is a polynomial of degree at least $e d$ in $x_{1}, \ldots, x_{d}$. That is, we can write

$$
s=w_{0}+\sum_{\ell=1}^{t} g_{\ell n}\left(x_{i}^{1 / p^{n}}\right) \phi_{\ell}\left(x_{i}^{1 / p^{n}}\right)
$$

for some $w_{0}$ that is a sum of terms each of which has a factor which is a monomial in $x_{1}, \ldots, x_{d}$ of degree $d e$ and the $\phi_{\ell}$ are some homogeneous polynomials. If we now recall that the degree of $g_{\ell}$ is $m_{\ell} e$, we have

$$
a_{1}^{\beta} r \# s=a_{1}^{\beta} r \# w_{0}+\sum_{\ell=1}^{t}\left(a_{1}^{m_{\ell} / p^{n}} \# g_{\ell n}\left(x_{i}^{1 / p^{n}}\right)\right)\left(a_{1}^{\beta-m_{\ell} / p^{n}} r \# \phi_{\ell}\left(x_{i}^{1 / p^{n}}\right)\right) .
$$

It then follows from Proposition 4, where the $a_{i}$ and $x_{i}$ are replaced with their $p^{n}$ th roots and the $n$ and $e$ of that proposition are $p^{n}$ and $e / p^{n}$, that each of the terms of $w_{0}$ is in the ideal $\left(a_{1} x_{1}, a_{2} x_{1}+\right.$ $\left.a_{1} x_{2}, \ldots, a_{d} x_{1}+\cdots+a_{1} x_{d}\right)$. If we take this expression modulo $p^{1 / p^{n}} C$, the factors $a_{1}^{m_{\ell} / p^{n}} \# g_{\ell n}\left(x_{i}^{1 / p^{n}}\right)$ are the leading terms of the $f_{\ell n}$ derived from $f_{\ell}$ as in section 4 . Thus condition 2 of the conditions on $\mu$ is satisfied.

To show that the third condition is also satisfied, let

$$
\sum_{j \geq 0} h_{\ell j n} P^{j / p^{n+m}}
$$


be the $n$th polynomial derived from $f_{\ell}$ as in section 4 . Since $f_{\ell}$ is of the form $a_{1}^{m_{\ell}} \# g_{\ell}$, it follows from Proposition 1 that each $h_{\ell j n}$ is of the form $a_{1}^{m_{\ell} / p^{n}} \# t_{\ell j n}$ for some $t_{\ell j n}$. We are taking the sum

$$
\sum_{\ell=1}^{t}\left(\sum_{j \geq 0} a_{1}^{m_{\ell} / p^{n}} \# t_{\ell j n} P^{j / p^{n+m}}\right)\left(a_{1}^{\beta-m_{\ell} / p^{n}} r \# \phi_{\ell}\left(x_{i}^{1 / p^{n}}\right)\right) .
$$

If $c_{j}$ is the coefficient of $P^{j / p^{n+m}}$ in this product for some $j$, then $c_{j}$ is a sum of elements of the form $\left(a_{1}^{m_{\ell} / p^{n}} \# s_{1}\right)\left(a_{1}^{\beta-m_{\ell} / p^{n}} r \# s_{2}\right)=a_{1}^{\beta} r \# s_{1} s_{2}$ for some $s_{1}$ and $s_{2}$, so $\mu\left(c_{j}\right) \geq d+\delta$. Thus condition three is satisfied.

\section{REFERENCES}

[1] S. Goto and K.-i. Watanabe, On graded rings. I, J. Math. Soc. Japan 30 (1978), 179-213.

[2] R. C. Heitmann, The direct summand conjecture in dimension three, Ann. of Math. (2) 156 (2002), 695-712.

[3] M. Hochster, Topics in the homological theory of modules over commutative rings, CBMS Regional Conference Series in Mathematics 24, 1975.

[4] M. Hochster and C. Huneke, Infinite integral extensions and big Cohen-Macaulay algebras, Ann. of Math. (2) 135 (1992), 53-89.

[5] C. Huneke and G. Lyubeznik, Absolute integral closure in positive characteristic, Advances Math. 210 (2007) $498-504$.

[6] H. Matsumura, Commutative Rings, Cambridge University Press, Cambridge, England (1986).

[7] P. Roberts, Intersection Theorems, in Commutative Algebra, Mathematical Sciences Research Institute Publications 15, Springer-Verlag (1989), pp. 417-436.

[8] P. Roberts, Fontaine rings and local cohomology, J. Algebra 323 (2010), 2257-2269.

[9] P. Roberts, The root closure of a ring of mixed characteristic, arXiv:0810.0215v1 [math.AC].

[10] P. Roberts, A. Singh, and V. Srinivas, Annihilators of local cohomology in characteristic zero, Ill. J. Math. 51 (2007), $237-254$. 